Pacific Journal of Mathematics

AMPLENESS IN COMPLEX HOMOGENEOUS SPACES AND A 


\title{
AMPLENESS IN COMPLEX HOMOGENEOUS SPACES AND A SECOND LEFSCHETZ THEOREM
}

\author{
NORMAN GOLDSTEIN
}

\begin{abstract}
This paper investigates how ampleness of the normal bundle of a smooth subvariety $Y$ of a complex homogeneous space $Z=G / H$ influences the intersection of $Y$ with other subvarieties of $Z$.
\end{abstract}

We consider a class of homogeneous spaces, rigged spaces, that includes Grassmannians, quadrics and $\mathbf{P}^{r} \backslash \mathbf{P}^{k}$ (the compliment in $\mathbf{P}^{r}$ of a linear subspace $\mathbf{P}^{k}$ ). A result of Corollary 4.5.2 is:

Let $Z$ be a rigged homogeneous space with group $G$. Let $Y$ be a compact smooth subvariety of $Z$ possessing an ample normal bundle $N Y$. (See [10] for the definition of ample.) Then the map

$$
\phi_{Y}: \mathbf{P}\left(N^{*} Y\right) \rightarrow \mathbf{P}^{a}
$$

determined by the $G$-sections of $T Z$ is generically $1-1$ (see 2.2 for the definition of $\phi_{Y}$ ).

Corollary 4.5.2 and Theorem 5.2 imply that if $X$ and $Y$ are both smooth and compact subvarieties of $Z$ with ample normal bundles, then for all $g \in G$, except for a closed codimension 2 subvariety of $G, X \cap$ $g^{-1}(Y)$ is either a transverse intersection, or has precisely one singular point and it is non-degenerate quadratic.

In $\$ 5$ these results are used to prove a generalized "second Lefschetz theorem on hyperplane sections", in analogy to the author's previous paper [6], and following the generalized first Lefschetz theorems of Barth $[2,2 \mathrm{~A}]$ and Sommese $[19,20]$.

I expand, now, the outline of the paper.

Section 1 begins by considering a holomorphic bundle map $\psi: E \rightarrow F$ of holomorphic vector bundles over a complex space $W$, i.e. $\psi_{x}: E_{x} \rightarrow F_{x}$ is linear for all $x \in W$. The linear fibre space $\mathcal{E}$ (see 4.1) is of central importance to the paper, and is defined as the $\operatorname{kernel} \operatorname{ker}\left(g^{*}\right):=g^{*-1}$ (zero section of $F$ ) for a certain bundle map $g^{*}$. (The confusing notation " $g$ " " for the bundle map does not refer, of course, to any one element $g \in G$ !) The map $g^{*}$ fits into a commutative diagram of vector bundles (4.2.3) and the results of Lemma 1.4 allow us to conclude, by a vector 
bundle "diagram chase", that $\mathcal{E}$ is isomorphic to $\operatorname{ker}\left(p^{*}\right)$, another linear fibre space.

Section 2 describes the ampleness map

$$
\phi: \mathbf{P}\left(T^{*} Z\right) \rightarrow \mathbf{P}\left(T_{e}^{*} G\right),
$$

and the orbit decomposition $\mathbf{P}\left(T^{*} Z\right)=\cup_{\rho} O_{\rho}$ under the action of $G$.

Section 3 defines "rigged spaces", and discusses why Grassmannians and quadrics are rigged.

Sections 4 and 5 , as already described, analyse the linear fibre space $\mathcal{E}$ and prove the Lefschetz theorem.

I would like to thank Andrew Sommese for many helpful and guiding conversations, and the Natural Sciences and Engineering Research Council Canada for their continuing support. Also, Jim Carrell has clarified for me many aspects of group actions.

1. Preliminaries. More background material is in [6, §0]. All mappings in this paper are regular, i.e., everywhere defined.

Let $X$ be a complex space. The reduction of $X$ is $X_{\text {red }}$, and we denote

$$
\operatorname{redim}(X)=\operatorname{dim}\left(X_{\text {red }}\right) .
$$

Let $\pi: E \rightarrow X$ be a complex vector bundle over the space $X$. We denote, also by " $X$ ", the zero section of $E$.

Let $\phi: E \rightarrow F$ be a linear map of vector bundles over $X$. Then $\phi^{-1}(X)=\operatorname{Ker} \phi$ is a linear fibre space over $X$ cf [5] (1.6).

Note. The variety $\operatorname{Ker}(\phi)$ is smooth at $e$ precisely when $\phi$ meets the zero section of $F$ transversely from $e$.

(1.1.2) Definition. Let $\pi: E \rightarrow X$ be a vector bundle over $X$. A local projection on $E$ is a map

$$
\nu: \pi^{-1}(U) \rightarrow \mathbf{C}^{b}
$$

where $U$ is a neighbourhood of $x \in X, \pi^{-1}(U) \simeq U \times \mathbf{C}^{b}$, and $\nu$ is the corresponding natural projection.

I omit the elementary proofs of the following four lemmas. 
(1.2) Lemma. Let $\phi: E \rightarrow F$ be a map of vector bundles over $X$, and suppose that $\phi(e) \in X$.

Then $\phi$ meets the zero section, $X$, of $F$ transversely from $e \Leftrightarrow \nu \circ \phi$ is a submersion for a local projection, $\nu$, on $F$ near $\phi(e)$.

(1.3) Lemma. Let $\phi: E \rightarrow F$ and $\psi: G \rightarrow F$ be maps of vector bundles over $X$. Suppose that $\phi(e)=\psi(g)$.

Then $\phi$ and $\psi$ meet transversely, from $e$ and $g$, respectively $\Leftrightarrow$ the map

$$
\phi \oplus(-\psi): E \oplus G \rightarrow F
$$

meets $X$ transversely from $(e, g)$.

(1.4) Lemma (i). Let $\phi: E \rightarrow F$ be a map of vector bundles over $X$. Then

$$
\phi: X \rightarrow X
$$

is an isomorphism.

(ii) Let $E \stackrel{\phi}{\rightarrow} F \stackrel{\psi}{\rightarrow} G$ be an exact sequence of vector bundles over $X$. Then $\phi(E) \subset \operatorname{Ker}(\psi)$, and

$$
\phi: E \rightarrow \operatorname{Ker}(\psi) \text { is a submersion, }
$$

i.e. the sequence is "exact" when viewed as a map of varieties.

(1.5) Lemma. Let $\mathcal{E}$ be a linear fibre space over $X$, and $\mathbf{P}(\mathcal{E})=$ $(\mathcal{E} \backslash X) / \mathrm{C}^{*}$ its projectivization. Let $e \in \mathcal{E}$ be non-zero.

Then $\mathcal{E}$ is smooth at $e$

$$
\Leftrightarrow \mathbf{P}(\mathcal{E}) \text { is smooth at }[e] \text {. }
$$

(1.6) LemMa. Let $f: X^{m} \rightarrow Y^{n}$ be a map of complex manifolds, and

$$
f^{*}: f^{-1}\left(T^{*} Y\right) \rightarrow T^{*} X
$$

the codifferential of $f$.

Then, as a map of varieties, the natural map

$$
f^{\prime}: \operatorname{Ker}\left(f^{*}\right) \rightarrow T^{*} Y
$$

has constant rank $n$. (See example below.)

Proof. Locally,

$$
\begin{aligned}
& T^{*} X=X \times \mathbf{C}^{m}, \\
& T^{*} Y=Y \times \mathbf{C}^{n},
\end{aligned}
$$




$$
\begin{aligned}
f^{-1}\left(T^{*} Y\right) & =X \times \mathbf{C}^{n}, \\
f^{*}(x, w) & =\left(x,\left(\frac{\partial f}{\partial x}(x)\right)^{t} w\right), \text { and } \\
\operatorname{Ker} f^{*} & =\left\{(x, w) \in X \times \mathbf{C}^{n}=w^{t} \frac{\partial f}{\partial x}(x)=0\right\} .
\end{aligned}
$$

Let $A$ be the $m \times(m+n)$ matrix

$$
\left(\frac{\partial^{2}\left(w^{t} f\right)}{\partial x^{2}}(x) \vdots\left(\frac{\partial f}{\partial x}(x)\right)^{t}\right)
$$

which is the Jacobean of the equations describing $\operatorname{Ker} f^{*}$ in $X \times \mathbf{C}^{n}$. Then

$$
\begin{aligned}
& T_{(x, w)}\left(\operatorname{Ker} f^{*}\right)=\operatorname{ker} A \\
& \quad=\left\{(u, v) \in \mathbf{C}^{m} \times \mathbf{C}^{n}: \frac{\partial^{2}\left(w^{t} f\right)}{\partial x^{2}}(x) u+\left(\frac{\partial f}{\partial x}(x)\right)^{t} v=0\right\} .
\end{aligned}
$$

Now, $f^{\prime}(x, w)=(f(x), w)$. It follows that

$$
\operatorname{ker} f_{*}^{\prime}=\left(\operatorname{ker} A^{t}\right) \oplus\{0\} .
$$

Thus

$$
\begin{aligned}
\mathrm{rk} f^{\prime} & =\operatorname{dim}(\operatorname{ker} A)-\operatorname{dim}\left(\operatorname{ker} A^{t}\right) \\
& =m+n-\mathrm{rk} A-(m-\mathrm{rk} A)=n .
\end{aligned}
$$

EXAMPLE. The above lemma may be illustrated with the map

$$
\begin{gathered}
f: X=\mathbf{C} \rightarrow Y=\mathbf{C}, \\
x \mapsto x^{2} ; \\
f^{*}: f^{-1}\left(T^{*} Y\right)=\mathbf{C}^{2} \rightarrow T^{*} X=\mathbf{C}^{2}, \\
\quad(x, w) \mapsto(x, 2 x w) ; \\
\operatorname{Ker} f^{*}=\left\{(x, w) \in \mathbf{C}^{2}: x w=0\right\} ; \\
f^{\prime}: \operatorname{Ker} f^{*} \rightarrow T^{*} Y=\mathbf{C}^{2}, \\
(x, w) \mapsto\left(x^{2}, w\right) .
\end{gathered}
$$

It is easy to compute that the differential of $f^{\prime}$ has constant rank 1 .

(1.7) LEMMA. Let

$$
f_{i}: X_{\iota} \rightarrow Y, \quad i=1, \ldots, a,
$$

be maps of complex algebraic varieties, and let $Y_{k}=\left\{y \in Y: \exists x_{i} \in X_{i}\right.$, $i=1, \ldots, a$ with $f_{i}\left(x_{i}\right)=y$ and $\left.\operatorname{dim}\left(\bigcap_{i=1} f_{i *}\left(T_{x_{i}}\left(X_{l}\right)\right)\right) \leq k\right\}$. 
Then $Y_{k}$ is a constructable subset of $Y$ of dimension at most $k$.

Proof (sketch). Let $\mathcal{X}$ be the fibre product of the $f_{i}$, and

$$
f: \mathfrak{X} \rightarrow Y
$$

the natural projection.

Then

$$
f_{*}\left(T_{\left(x_{1}, \ldots, x_{a}\right)} \mathfrak{X}\right)=\bigcap_{i=1}^{a} f_{i *}\left(T_{x_{i}} X_{i}\right) .
$$

Thus,

$$
Y_{k}=f(\{r k(f) \leq k\})
$$

and the lemma follows.

(1.7.1) Corollary. Let $f: X \rightarrow Y$ be a map of complex algebraic varieties. Let

$$
Y_{k}=\left\{y \in Y: \operatorname{dim}\left(\bigcap_{x \in f^{-1}(y)} f_{*}\left(T_{x} X\right)\right) \leq k\right\} .
$$

Then $Y_{k}$ is a constructable subset of $Y$ of dimension at most $k$.

Proof. Let $a$ be the local embedding dimension of $Y$, and apply the lemma to $a$ copies of the map $f$.

Let $Z$ be a complex algebraic homogeneous space with algebraic group $G$. The evaluation map

$$
\begin{aligned}
Z \times G & \rightarrow Z, \\
(z, g) & \rightarrow g z
\end{aligned}
$$

is necessarily algebraic (cf [3], Proposition 7).

Let $X$ and $Y$ be Zariski locally closed smooth subvarieties of $Z$. The main tool that we use is the family of intersections

$$
\left\{X \cap g^{-1}(Y)\right\}_{g \in G} \text {. }
$$

Let

$$
\mu: X \times G \rightarrow Z
$$

be the restriction of the evaluation (1.8) to $X \times G$. Then $\eta=\mu^{-1}(Y)$ is a Zariski locally closed smooth subvariety of $X \times G$. 


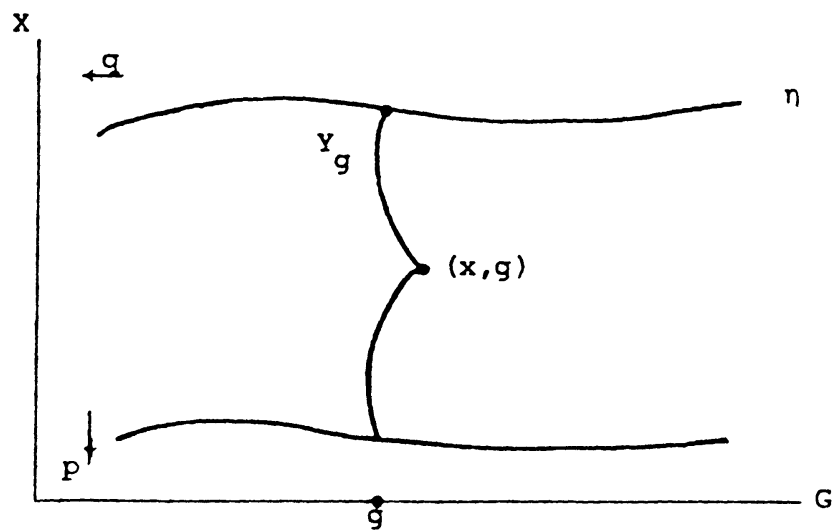

Let $p$ and $q$ be the restrictions to $\eta$ of the natural projections on $X \times G$, as in Figure (1.10). Let $Y_{g}$ denote the variety $X \cap g^{-1}(Y)$. Then

$$
Y_{g} \simeq p^{-1}(g) \text { via } q \text {. }
$$

For simplicity, we assume that $Z, G, X$ and $Y$ are connected. Let $\operatorname{dim} Z=r, \operatorname{dim} G=N, \operatorname{dim} X=n$ and $\operatorname{dim} Y=r-s$. Let

$$
\operatorname{cork}_{x}\left(Y_{g}\right)=\operatorname{dim}\left(T_{x}\left(Y_{g}\right)\right)-(n-s)
$$

be the corank of $Y_{g}$ at $x$. Then $Y_{g}$ is smooth at $x$ (i.e. $X$ meets $g^{-1}(Y)$ transversely at $x$ ) precisely when $\operatorname{cork}_{x}\left(Y_{g}\right)=0$. Each smooth $Y_{g}$ has pure dimension $n-s$. Moreover

$$
\operatorname{rank}_{(x, g)} p=N-\operatorname{cork}_{x}\left(Y_{g}\right) .
$$

Let

$$
\mathcal{S}=\left\{(x, g) \in \eta: x \text { is a singular point of } Y_{g}\right\} .
$$

2. Ampleness and group actions. In [17] $\S 1$, Sommese defines the notion of $k$-ampleness:

Let $A$ be a reduced compact complex space. A line bundle $L \rightarrow A$ is $k$-ample if the sections of some tensor power of $L$ induce a map of $A$ to projective space, whose fibre dimensions are at most $k$. Ample is the same as 0 -ample. A vector bundle $E \rightarrow A$ is $k$-ample if the tautological line bundle

$$
O_{\mathbf{P}\left(E^{*}\right)}(1) \rightarrow \mathbf{P}\left(E^{*}\right)
$$

is $k$-ample. Here, $\mathbf{P}\left(E^{*}\right)=\left(E^{*} \backslash A\right) / \mathbf{C}^{*}$. 
If $E$ is spanned by sections $\left\{s_{0}, \ldots, s_{a}\right\}$, it follows that $E$ is $k$-ample precisely when the map

$$
\mathbf{P}\left(E^{*}\right) \rightarrow \mathbf{P}^{a}
$$

defined by $\left\{s_{0}, \ldots, s_{a}\right\}$ has maximal fibre dimension at most $k$.

Let $Z$ and $G$ be as in (1.8), and let $e$ be the identity element of $G$. Each $v \in T_{e} G$ determines a section

$$
\begin{aligned}
S_{v}: Z & \rightarrow T Z, \\
z & \mapsto z_{*}^{\#}(v) \in T_{z} Z,
\end{aligned}
$$

where

$$
\begin{aligned}
z^{\#}: G & \rightarrow Z, \\
g & \mapsto g z .
\end{aligned}
$$

These sections span $T Z$ since $z^{\#}$ is a submersion. Let $v_{1}, \ldots, v_{N}$ be a basis of $T_{e} G$. The sections $\left\{S_{v_{1}}, \ldots, S_{v_{N}}\right\}$ induce the map

$$
\begin{aligned}
& \phi: T^{*} Z \rightarrow \mathbf{C}^{N} \\
& \begin{aligned}
T_{z}^{*} Z \ni \alpha \mapsto( & \left.\alpha\left(S_{v_{1}}(z)\right), \ldots, \alpha\left(S_{v_{N}}(z)\right)\right) \\
& =\left(z^{\# *}(\alpha)\left(v_{1}\right), \ldots, z^{\# *}(\alpha)\left(v_{N}\right)\right) .
\end{aligned}
\end{aligned}
$$

Thus, $\phi$ may be viewed in the coordinate free way

$$
\begin{aligned}
& \phi: T^{*} Z \rightarrow T_{e}^{*} G, \\
& T_{z}^{*} Z \ni \alpha \mapsto z^{\# *}(\alpha) .
\end{aligned}
$$

We denote, also by “ $\phi$ ", the map

$$
\phi: \mathbf{P}\left(T^{*} Z\right) \rightarrow \mathbf{P}\left(T_{e}^{*} G\right)
$$

Let $Y$ be a smooth subvariety of $Z$, and $N Y$ the normal bundle of $Y$ in $Z$. Then, $S_{v_{1}}, \ldots, S_{v_{N}}$ induce sections which span $N Y$, and

$$
\phi_{Y}=\left.\phi\right|_{N^{*} Y}: N^{*} Y \rightarrow T_{e}^{*} G
$$

is the map that they define. If $Y$ is compact, then $N Y$ is $k$-ample precisely when

$$
\phi_{Y}: \mathbf{P}\left(N^{*} Y\right) \rightarrow \mathbf{P}\left(T_{e}^{*} G\right)
$$

has fibre dimensions at most $k$.

Group Actions. Let $Z$ be a complex algebraic homogeneous space, as in (1.8). The action of $G$ on $Z$ induces an action of $G$ on $T^{*} Z$, through 
linear maps, and hence an algebraic action

$$
\mathbf{P}\left(T^{*} Z\right) \times G \rightarrow \mathbf{P}\left(T^{*} Z\right) .
$$

Let

$$
\mathbf{P}\left(T^{*} Z\right)=\bigcup_{\rho} O_{\rho}
$$

be the orbit decomposition of the action (2.3). Each orbit $O_{\rho}$ is an irreducible, Zariski locally closed, smooth subvariety of $\mathbf{P}\left(T^{*} Z\right)$. The natural projection $\mathbf{P}\left(T^{*} Z\right) \rightarrow Z$ is $G$-equivariant. Since the action of $G$ on $Z$ is transitive, there is a 1-1 correspondence between orbits of $G$ in $\mathbf{P}\left(T^{*} Z\right)$ and orbits of $G_{z}$ in $\mathbf{P}\left(T_{z}^{*} Z\right)$, where $G_{z}$ is the isotropy group of an element $z \in Z$.

Let $Y$ be a Zariski locally closed, smooth subvariety of $Z$. Then, $\mathbf{P}\left(N^{*} Y\right) \subset \mathbf{P}\left(T^{*} Z\right)$, and we define

$$
\begin{aligned}
\mathbf{P}\left(N^{*} Y\right)_{\rho} & =\mathbf{P}\left(N^{*} Y\right) \cap O_{\rho}, \\
d_{\rho}^{Y} & =\operatorname{redim}\left(\mathbf{P}\left(N^{*} Y\right)_{\rho}\right)
\end{aligned}
$$

In particular, let

$$
d_{\rho}=d_{\rho}^{\{z\}}=\text { dimension of the } \rho \text { th orbit }
$$

of the action of the isotropy group $G_{z}$ on $\mathbf{P}\left(T_{z}^{*} Z\right)$.

It is easily seen that

$$
\operatorname{dim}\left(O_{\rho}\right)=r+d_{\rho}
$$

where $\operatorname{dim} Z=r$.

3. Rigged spaces. This paper is concerned, ostensibly, with Grassmannians and quadrics. In this section, we define a class of homogeneous spaces, rigged spaces, which includes the above two families. For simplicity, in the remainder of the paper, we will be assuming that the ambient homogeneous space, $Z$, is rigged, although it will be clear that many of the results are true under less stringent hypotheses.

(3.1) Definition. Let $Z$ be a complex algebraic homogeneous space with connected group $G$. We say that $Z$ is rigged if

(i) The universal covering map of $G$ is algebraic. As is well-known, this is equivalent to $\pi_{1}(G)$ being finite, and this is the case when $G$ is semisimple. 
(ii) There are finitely many orbits $O_{1}, \ldots, O_{m}$ for the action of $G$ on $\mathbf{P}\left(T^{*} Z\right)$ cf (2.3). In particular, there is a (unique) open orbit, say, $O_{m}$.

(iii) The isotropy group of an element of $O_{m}$ is connected.

(iv) The map

$$
\phi: \mathbf{P}\left(T^{*} Z\right) \rightarrow \mathbf{P}\left(T_{e}^{*} G\right)
$$

is $1-1$ on $O_{m} \mathrm{cf}(2.1)$. This condition is equivalent to:

(iv') The isotropy group of an element $\alpha$ of $O_{m}$ is equal to the isotropy group of $\phi(\alpha)$ with respect to the co-adjoint action of $G$ on $\mathbf{P}\left(T_{e}^{*}(G)\right)$.

(v) For each orbit $O_{\rho}$, there is a Zariski locally closed submanifold $X_{\rho}$ of $Z$ such that $\mathbf{P}\left(N^{*}\left(X_{\rho}\right)\right)$ is generically contained in $O_{\rho}$ i.e. $d_{\rho}^{X_{\rho}}=r-1$, cf. (2.4).

We discuss, now, briefly, why Grassmannians and quadrics are rigged, and mention only that $\mathbf{P}^{r} \backslash \mathbf{P}^{k}$ is rigged where $\mathbf{P}^{k}$ is a linear projective $k$-subspace of $\mathbf{P}^{r}$, and the group $G$ is $\left\{g \in \operatorname{PGL}(r, \mathbf{C}): g\left(\mathbf{P}^{k}\right)=\mathbf{P}^{k}\right\}$.

Grassmannians. Let $Z=\operatorname{Gr}\left(t, \mathbf{C}^{\tau+t}\right)$ be the Grassmann manifold of $\mathbf{C}^{t}$ 's in $\mathbf{C}^{\tau+t}$. The group $G$ is the projective linear group $\operatorname{PGL}(\tau+t-1, \mathbf{C})$. The universal cover of $G$ is the algebraic projection

$$
\operatorname{SL}(\tau+t, \mathbf{C}) \rightarrow G
$$

where $\operatorname{SL}(\tau+t, \mathbf{C})$ is the special linear group in $\mathbf{C}^{\tau+t}$.

Over $Z$, there is a well known tautological sequence

$$
0 \rightarrow E \stackrel{i}{\rightarrow} O^{\tau+t} \stackrel{\pi}{\rightarrow} F \rightarrow 0
$$

where $E$ and $F$ are, respectively, rank $t$ and rank $\tau$ vector bundles. Moreover,

$$
T^{*} Z=\operatorname{Hom}(F, E)
$$

so that each element $\alpha \in T_{z}^{*} Z$ has a rank between 0 and $m=\min (\tau, t)$ its rank as a linear map

$$
\alpha: F_{z} \rightarrow E_{z}
$$

We can write

$$
\mathbf{P}\left(T^{*} Z\right)=\bigcup_{\rho=1}^{m} O_{\rho}
$$

where $O_{\rho}$ is the projectivization of the elements of rank $\rho$. This is also the orbit decomposition of the action of $G$ on $\mathbf{P}\left(T^{*} Z\right)$. The orbit $O_{m}$ of the maximal rank vectors is the open orbit. A calculation shows that the 
isotropy groups are connected, and that

$$
d_{\rho}=t \tau-(t-\rho)(\tau-\rho)-1, \quad \text { where } t \tau=r=\operatorname{dim} Z .
$$

Consider now the map

$$
\begin{aligned}
& \phi: \mathbf{P}\left(T^{*} Z\right) \rightarrow \mathbf{P}\left(T_{e}^{*} G\right) \\
& \phi(\alpha)=\text { projectivization of } i_{z} \circ \alpha \circ \pi_{z} .
\end{aligned}
$$

Here, $T_{e}^{*} G$ is identified with $\operatorname{sl}(\tau+t, \mathbf{C}), i_{z}$ is the inclusion $E_{z} \rightarrow \mathbf{C}^{\tau+t}$ and $\pi_{z}$ is the projection $\mathbf{C}^{\tau+t} \rightarrow F_{z}$. From this, one sees that $\operatorname{rk} \alpha=\operatorname{rk} \phi(\alpha)$. Let, now, $A=\phi(\alpha)$ have $\operatorname{rank} \rho$. Then $\phi^{-1}(A) \simeq\{z \in Z: \operatorname{im} A \subset z \subset \operatorname{ker} A\}$ $\simeq \operatorname{Gr}\left(t-\rho, \mathbf{C}^{\tau+t-2 \rho}\right)$. In particular, $\phi$ is $1-1$ on $O_{m}$.

Finally, let $\mathbf{C}^{\tau+t-\rho}$ be a codimension $\rho$ subspace of $\mathbf{C}^{\tau+t}$, and put

$$
X_{\rho}=\left\{z \in Z: z \subset \mathbf{C}^{t+\tau-\rho}\right\} .
$$

Then $\mathbf{P}\left(N^{*} X_{\rho}\right)$ is generically contained in $O_{\rho}$.

Quadrics. Let $Z=2^{r} \subset \mathbf{P}^{r+1}$ be the $r$-dimensional quadric. The group $G$ is the projectivization of the special complex orthogonal group $\mathbf{P}(\mathrm{SO}(r+2, \mathbf{C}))$ and has a finite fundamental group (of order 2 for $r$ odd and order 4 for $r$ even).

There is a commutative diagram

$$
\begin{aligned}
& \mathbf{P}\left(T^{*} Z\right) \stackrel{\phi}{\rightarrow} \mathbf{P}\left(T_{e}^{*} G\right) \\
& \Gamma \downarrow \quad \nearrow \text { Plücker embedding } \\
& \operatorname{Gr}\left(1, \mathbf{P}^{r+1}\right)
\end{aligned}
$$

The image of $\Gamma$ consists of all $\mathbf{P}^{1}$,s that are tangent to $Z$. If $\alpha \in \mathbf{P}\left(T^{*} Z\right)$, then

$$
\Gamma^{-1}(\Gamma(\alpha)) \simeq \Gamma(\alpha) \cap Z= \begin{cases}\mathbf{P}^{1}, & \Gamma(\alpha) \subset Z \\ \text { point, } & \Gamma(\alpha) \not \subset Z .\end{cases}
$$

Now, $\mathbf{P}\left(T^{*} Z\right)=O_{1} \cup O_{m}$ is the orbit decomposition where

$$
\begin{aligned}
& O_{1}=\left\{\alpha \in \mathbf{P}\left(T^{*} Z\right): \Gamma(\alpha) \subset Z\right\} \quad \text { and } \\
& O_{m}=\left\{\alpha \in \mathbf{P}\left(T^{*} Z\right): \Gamma(\alpha) \not \subset Z\right\} .
\end{aligned}
$$

In particular, $\phi$ is $1-1$ on $O_{m}$. Again, a computation shows that the isotropy groups are connected, and $d_{1}=r-2$. 
Finally, let $X_{1}$ be the smooth points of a singular hyperplane section of $Z$. Then, $\mathbf{P}\left(N^{*}\left(X_{1}\right)\right)$ is contained in $O_{1}$.

4. Analytical results on rigged spaces. Throughout this section, we study the relationship between two smooth subvarieties $X$ and $Y$ of the homogeneous space $Z$. The main tool that we use is a linear fibre space $\mathcal{E}$ which contains information of how singular are the intersections of $X$ with the translates of $Y$ by the group $G$.

(4.1) The Construction of $\mathcal{E}$. Recall the maps

$$
\begin{array}{ll}
\mu: \eta \rightarrow Y, & q: \eta \rightarrow X, \\
(x, g) \mapsto g x, & (x, g) \mapsto x .
\end{array}
$$

Define a map (with abuse of notation)

$$
\begin{gathered}
g^{*}: \mu^{-1}\left(N^{*} Y\right) \rightarrow q^{-1}\left(T^{*} X\right), \\
N_{g x}^{*} Y \ni \alpha \mapsto g^{*}(\alpha) \in T_{x}^{*} X
\end{gathered}
$$

and let

$$
\mathcal{E}=\operatorname{Ker}\left(g^{*}\right)
$$

cf. $§ 1$. We note that

$$
\mathcal{E}_{(x, g)}=g^{*-1}\left(N_{x}^{*} X\right) \cap N_{g x}^{*} Y=\left(g_{*}\left(T_{x} X\right)+T_{g x} Y\right)^{\perp} \subset T_{g x}^{*} Z
$$

In particular

$$
\operatorname{dim}\left(\mathcal{E}_{(x, g)}\right)=\operatorname{cork}_{x}\left(Y_{g}\right) .
$$

(4.2) Bounding $\mathcal{E}$. Let $\mathbf{P}(\mathcal{E})=(\mathscr{E} \backslash \eta) / \mathbf{C}^{*}$ be the projectivization of $\tilde{E}$. The map $\mathcal{E} \rightarrow \eta$ induces the map $\mathbf{P}(\mathcal{E}) \rightarrow \mathcal{S}$. Also, the map $\phi_{Y}: N^{*} Y \rightarrow T_{e}^{*} G$ induces the map

$$
\begin{array}{r}
\phi_{\mathscr{E}}: \mathcal{G} \rightarrow G \times T_{e}^{*} G, \\
\mathcal{E}_{(x, g)} \ni \alpha \mapsto\left(g, \phi_{Y}(\alpha)\right) .
\end{array}
$$

LEMMA. There is a commutative diagram

$$
\begin{array}{ccc}
\mathcal{E} & \stackrel{\phi_{\mathcal{G}}}{\rightarrow} & G \times T_{e}^{*} G \\
\imath \downarrow & & \imath \downarrow \\
\operatorname{Ker}\left(p^{*}\right) & \stackrel{p^{\prime}}{\rightarrow} & T^{*} G
\end{array}
$$

(See (1.6) for the definition of $p^{\prime}$.) The vertical maps are isomorphisms. 
Proof.

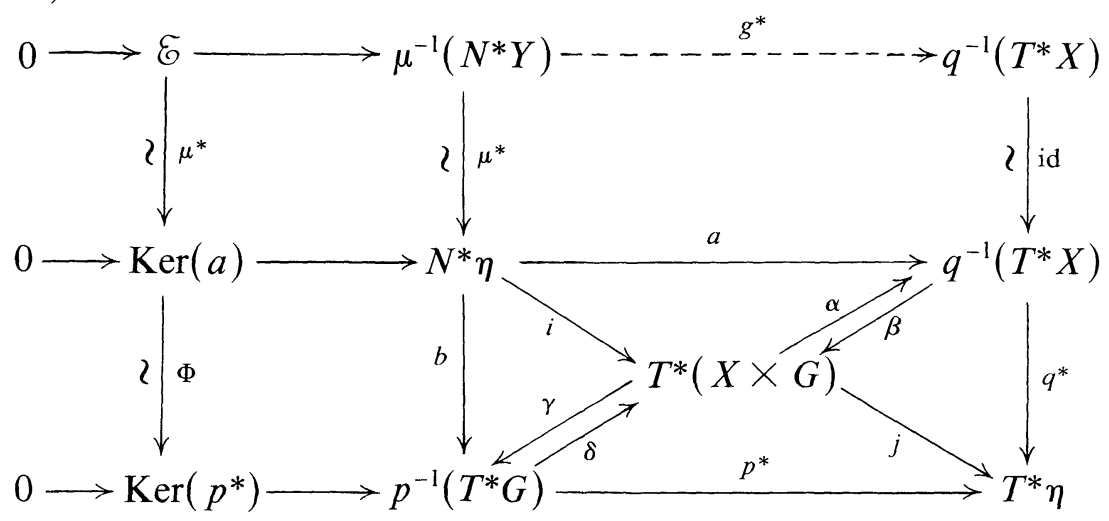

In Figure (4.2.3), one of the diagonal exact sequences is for the conormal bundle of $\eta$ in $X \times G$. The other expresses the direct sum decomposition of $T^{*}(X \times G)$. The maps $a$ and $b$ are defined, respectively, as $\alpha i$ and $\gamma i$. It is clear that $q^{*}=j \beta$ and $p^{*}=j \delta$. Since

$$
\beta \alpha+\delta \gamma=\mathrm{id}_{T^{*}(X \times G)}
$$

we have

$$
q^{*} a+p^{*} b=j i=0
$$

This, Lemma (1.4) and a diagram chase show that

$$
\Phi: \operatorname{Ker}(a) \rightarrow \operatorname{Ker}\left(p^{*}\right)
$$

is well-defined and an isomorphism of varieties.

As for the top two rows of Figure (4.2.3), the map $\mu: X \times G \rightarrow Z$ is a submersion and $\eta=\mu^{-1}(Y)$. Thus, by a dimension count, the codifferential of $\mu$ induces an isomorphism

$$
\mu^{*}: \mu^{-1}\left(N^{*} Y\right) \rightarrow N^{*} \eta
$$

The composition $a \circ \mu^{*}$ is just "the partial of $\mu$ with respect to $x$ " viz. $g^{*}$. This fills in the top row of Figure (4.2.3), inducing, also, the isomorphism

$$
\mu^{*}: \mathcal{E} \rightarrow \operatorname{Ker}(a) \text {. }
$$


To complete the proof of the lemma, the two left columns of Figure (4.2.3) give the diagram

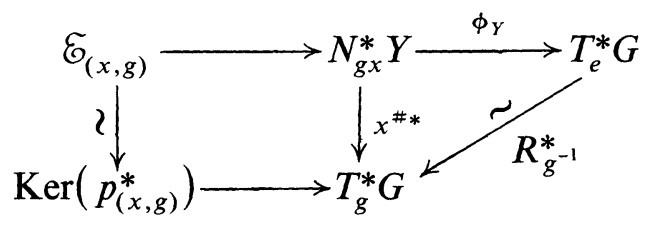

Here, we have replaced $b \circ \mu^{*}$ with $x^{\# *}$ i.e. "the partial of $\mu$ with respect to $g$ ". Now, $x^{\#}=(g x)^{\#} \circ R_{g}^{-1}$, so that $x^{\# *}=R_{g^{-1}}^{*} \circ \phi_{Y}$, cf. (2.2). This fills in the right half of Figure (4.2.4), and proves the lemma.

(4.3) Proposition. Let $X$ be a locally closed smooth subvariety of the complex algebraic homogeneous space $Z$. If $Y$ is a compact smooth subvariety of $Z$ and $N Y$ is $k$-ample, then

$$
\operatorname{redim}(\mathbf{P}(\varepsilon)) \leq N-1+k
$$

Proof. Projectivize diagram (4.2.2).

$$
\mathbf{P}(\mathcal{E}) \quad \stackrel{\phi_{\mathscr{E}}}{\rightarrow} G \times \mathbf{P}\left(T_{e}^{*} G\right)
$$

$$
\begin{array}{ccc}
\imath \downarrow & & \imath \downarrow \\
\mathbf{P}\left(\operatorname{Ker} p^{*}\right) & \stackrel{p^{\prime}}{\rightarrow} & \mathbf{P}\left(T^{*} G\right)
\end{array}
$$

By Lemma (1.6), rk $p^{\prime} \equiv N-1$.

Thus, redim(im $\left.\phi_{\mathscr{E}}\right) \leq N-1$. But, $Y$ is compact and $N Y k$-ample, so the fibres of $\phi_{Y}$ are at most $k$-dimensional. The same is true of $\phi_{\mathscr{E}}$, and it follows that

$$
\operatorname{redim}(\mathbf{P}(\mathcal{E})) \leq N-1+k .
$$

(4.4) Definition. We define the decomposition

$$
\begin{aligned}
\mathbf{P}(\mathscr{E}) & =\bigcup_{\rho=1}^{m} \mathbf{P}(\mathscr{E})_{\rho} \\
\text { by } \mathbf{P}(\mathscr{E}) & =\mathbf{P}(\mathscr{E}) \cap \mu^{-1}\left(\mathbf{P}\left(N^{*} Y\right)_{\rho}\right) \\
& =\left\{\alpha \in \mathbf{P}(\mathcal{E}): \alpha \text { is in the } \rho \text { th orbit } O_{\rho}\right\} .
\end{aligned}
$$

(4.5) Proposition. Let $Y$ be a smooth compact subvariety of the rigged homogeneous space $Z$. If $N Y$ is $k$-ample then the reduced dimension of each component of $\mathbf{P}\left(N^{*} Y\right)_{\rho}$ is between $d_{\rho}$ and $k+d_{\rho}$, cf. (2.4). 
Proof. A lower bound for the dimension of each component of $\mathbf{P}\left(N^{*} Y\right) \cap O_{\rho}$ is

$$
\begin{aligned}
& \operatorname{dim}\left(\mathbf{P}\left(N^{*} Y\right)\right)-\operatorname{cod}_{\mathbf{P}\left(T^{*} Z\right)} O_{\rho} \\
& =r-1-\left(2 r-1-\left(r+d_{\rho}\right)\right)=d_{\rho} .
\end{aligned}
$$

To determine an upper bound, let $X$ be any locally closed smooth subvariety of $Z$, and construct the space $\mathcal{E}$ for $X$ and $Y$. Define a map (cf. (4.1.2))

$$
\begin{gathered}
\theta: \mathbf{P}(\mathcal{E}) \rightarrow \mathbf{P}\left(N^{*} X\right) \times \mathbf{P}\left(N^{*} Y\right), \\
\mathbf{P}\left(\mathcal{E}_{(x, g)}\right) \ni \beta \mapsto\left(g^{*} \beta, \beta\right) .
\end{gathered}
$$

For each $\rho$,

$$
\mathbf{P}(\varepsilon)_{\rho}=\theta^{-1}\left(\mathbf{P}\left(N^{*} X\right)_{\rho} \times \mathbf{P}\left(N^{*} Y\right)_{\rho}\right) .
$$

Thus,

$$
\theta: \mathbf{P}(\mathcal{E})_{\rho} \rightarrow \mathbf{P}\left(N^{*} X\right)_{\rho} \times \mathbf{P}\left(N^{*} Y\right)_{\rho}
$$

and this restriction has fibres isomorphic to the isotropy group of an element of $O_{\rho}$, i.e. the fibres are $N-r-d_{\rho}$ dimensional.

By (4.3),

$$
k+N-1 \geq \operatorname{redim} \mathbf{P}(\mathcal{E})_{\rho}=d_{\rho}^{X}+d_{\rho}^{Y}+N-r-d_{\rho},
$$

so $d_{\rho}^{X}+d_{\rho}^{Y} \leq r-1+d_{\rho}+k$. But, since $Z$ is rigged, there is an $X$ with $d_{\rho}^{X}=r-1$.

Hence $d_{\rho}^{Y} \leq d_{\rho}+k$.

(4.5.2) CoRollary. Let $\kappa=r-2-\sup _{\rho \neq m}\left\{d_{\rho}\right\}$. If $Z$ is rigged, $Y$ compact, and $N Y$ is $\kappa$-ample, then $\mathbf{P}\left(N^{*} Y\right)$ meets the open orbit $O_{m}$, and

$$
\phi_{Y}: \mathbf{P}\left(N^{*} Y\right) \rightarrow \mathbf{P}\left(T_{e}^{*} G\right)
$$

is generically 1-1. For the Grassmannian $Z=\operatorname{Gr}\left(t, \mathbf{C}^{\tau+t}\right)$ we have $\kappa=$ $|t-\tau|$, and for quadrics $\kappa=0$.

Proof. The hypotheses ensure that $d_{\rho}^{Y} \leq r-2$ for $\rho \neq m$, so that $\mathbf{P}\left(N^{*} Y\right)_{m}$ must be non-empty. The definition of rigged implies that $\phi_{Y}$ is 1-1 on this Zariski open set. The specific values of $\kappa$ may be computed (cf. $\S 3)$.

(4.5.3) Corollary. Let $Z$ be rigged, $Y$ compact, $N Y$ ample, and assume that $\mathbf{P}\left(N^{*} X\right)$ meets the open orbit $O_{m}$. Then $\operatorname{redim}\left(\mathbf{P}(\mathscr{E})_{\rho}\right) \leq N-2$ for $\rho \neq m$. 
Proof. Using the map (4.5.1) we have

$$
\begin{aligned}
\operatorname{redim}\left(\mathbf{P}(\mathcal{E})_{\rho}\right) & =d_{\rho}^{X}+d_{\rho}^{Y}+N-r-d_{\rho} \\
& =d_{\rho}^{X}+N-r \leq N-2 \text { for } \rho \neq m \text { since } \mathbf{P}\left(N^{*} X\right)
\end{aligned}
$$

meets the open orbit $O_{m}$.

We fix, now, an element $z_{0}$ of $Z$.

(4.6) Note. Let $\sigma$ denote a local cross section of the bundle

$$
\begin{aligned}
z_{0}^{\#}: G & \rightarrow Z, \\
g & \mapsto g z_{0} .
\end{aligned}
$$

So, $\sigma_{z} \in G, \sigma_{z}\left(z_{O}\right)=z \forall z \in U=$ domain of $\sigma$, and $\sigma$ determines a local projection (cf. (1.1.2))

$$
\begin{gathered}
\nu:\left.\left(T^{*} Z\right)\right|_{U} \rightarrow T_{z_{O}}^{*} Z, \\
T_{z}^{*} Z \ni \alpha \mapsto \sigma_{z}^{*}(\alpha) .
\end{gathered}
$$

(4.7) Proposition. Let $X$ and $Y$ be smooth connected subvarieties of the rigged homogeneous space $Z$.

Then $\mathbf{P}(\mathcal{E})_{m}$ is a smooth $(N-1$ dimensional) connected open subvariety of $\mathbf{P}(\mathcal{E})$.

Proof. Certainly, $\mathbf{P}(\mathcal{E})_{m}$ is open, and the fibration (4.5.1) shows that $\mathbf{P}(\mathscr{E})_{m}$ is connected. By Lemma (1.5), we need only verify the smoothness of $\mathcal{E}$.

$$
\begin{array}{cccc}
0 \rightarrow \mathcal{E} \rightarrow & \mu^{-1}\left(N^{*} Y\right) & \stackrel{g^{*}}{\rightarrow} & q^{-1}\left(T^{*} X\right) \\
\downarrow & \downarrow g^{*} & & 2 \downarrow \text { id } \\
0 \rightarrow q^{-1}\left(N^{*} X\right) \rightarrow & q^{-1}\left(T^{*} Z\right) & \rightarrow & q^{-1}\left(T^{*} X\right)
\end{array}
$$

The top row of (4.7.1) defines $\mathcal{E}$ as $\operatorname{Ker}\left(g^{*}\right) \mathrm{cf}$. (4.1.1). In particular, $\mathcal{E}$ is smooth (i.e. $N$-dimensional) when the horizontal $g^{*}$ meets the zero section of $q^{-1}\left(T^{*} X\right)$ transversely. Now, by Lemma (1.4), $\mathcal{E}$ is smooth precisely when the vertical $g^{*}$ meets $q^{-1}\left(N^{*} X\right)$ transversely. By Lemma (1.3), $\mathscr{E}$ is smooth precisely when

$$
\begin{aligned}
\mu^{-1}\left(N^{*} Y\right) \oplus q^{-1}\left(N^{*} X\right) \rightarrow q^{-1}\left(T^{*} Z\right), \\
(\beta, \alpha)_{(x, g)} \mapsto\left(g^{*}(\beta)-\alpha\right)_{(x, g)} \in T_{x}^{*} Z
\end{aligned}
$$

meets the zero section transversely. 
Let $\nu$ be the local projection of $q^{-1}\left(T^{*} Z\right)$ defined in Note (4.6). Now, by Lemma (1.2), $\mathcal{E}$ is smooth precisely when

$$
\begin{aligned}
\mu^{-1}\left(N^{*} Y\right) \oplus q^{-1}\left(T^{*} X\right) \rightarrow T_{z_{0}}^{*} Z, \\
(\beta, \alpha)_{(x, g)} \mapsto \sigma_{x}^{*}\left(g^{*}(\beta)-\alpha\right)
\end{aligned}
$$

is a submersion.

Fix $\alpha, \beta, x$ and $y$, and consider $g$ 's of the form $g=\sigma_{y} h \sigma_{x}^{-1}$ where $h \in G_{z_{0}}$, the isotropy group of $z_{0}$. For (4.7.2) to be a submersion, it suffices that

$$
\begin{aligned}
\text { C }^{*} \times G_{z_{0}} & \rightarrow T_{z_{0}}^{*} Z, \\
(\lambda, h) & \rightarrow h^{*}\left(\sigma_{y}^{*}(\lambda \beta)\right)=\lambda h^{*} \sigma^{*} y(\beta)
\end{aligned}
$$

be a submersion, and this is equivalent to

$$
\begin{aligned}
G_{z_{0}} & \rightarrow \mathbf{P}\left(T_{z_{0}}^{*} Z\right), \\
h & \rightarrow h^{*} \sigma_{y}^{*}(\beta)
\end{aligned}
$$

being a submersion, which is certainly the case when $\beta$ is in the open orbit $O_{m}$.

5. A second Lefschetz theorem. In [6], a Second Lefschetz Theorem is discussed and proved in the context of complex projective space. This section proves an analogous theorem for rigged homogeneous spaces (cf. §3). The version of the First Leftschetz Theorem that we use is due to Sommese $[18,19,20]$ :

(5.0) Let $Z$ be a complex algebraic homogeneous space, $Y$ a smooth connected compact subvariety of $Z$ with an ample normal bundle, and $X$ a smooth connected closed subvariety of $Z$. Let

$$
\operatorname{dim} Z=r, \quad \operatorname{dim} X=n \text { and } \operatorname{dim} Y=r-s .
$$

Assume that $2 s \leq r$. Then

$$
\pi_{i}(X, X \cap Y, y)=0 \text { for } i \leq \min (n-s, r-2 s+1) .
$$

In particular, if $X=Z$ then

$$
\pi_{i}(Z, Y, y)=0 \text { for } i \leq r-2 s+1,
$$

or if $n+s \leq r+1$ then

$$
\pi_{i}(X, X \cap Y, y)=0 \text { for } i \leq n-s .
$$

(5.1) With the notation of (5.0), we recall (1.11) that

$$
\mathcal{S}=\left\{(x, g) \in \eta: x \text { is a singular point of } Y_{g}\right\}
$$

is a closed subvariety of $\eta$. 
Let $\delta_{\alpha}=\left\{(x, g) \in \eta: \operatorname{cork}_{x}\left(Y_{g}\right)=\alpha\right\}$ (cf. §1) so that $\delta=\cup_{\alpha \geq 1} \delta_{\alpha}$, and for each $k \cup_{\alpha \geq k} \delta_{\alpha}$ is a closed subvariety of $\mathcal{S}$. The space $\mathcal{S}_{1}$ possesses a natural variety structure, as defined in [6], (3.3). (The same is true of all the $\delta_{\alpha}$, but we will not be using them.)

Let $\Delta=p(\delta)$ be the discriminant of the family, i.e.,

$$
\Delta=\left\{g \in G: Y_{g} \text { is singular }\right\} .
$$

The hypotheses on $X$ and $Y$ ensure that $p$ is proper, so that $\Delta$ is a proper closed algebraic subvariety of $G$.

Fix a base point $o \in G \backslash \Delta$, and let

$$
\mathfrak{T C} \operatorname{Aut}\left(H_{n-s}\left(Y_{0}, \mathbf{C}\right)\right)
$$

be the monodromy group associated to the fibre bundle

$$
p: \eta \backslash p^{-1}(\Delta) \rightarrow G \backslash \Delta .
$$

We define the invariant cycles

$$
\text { inv }=\left\{v \in H_{n-s}\left(Y_{0}, \mathbf{C}\right): T v=v \forall T \in \mathscr{N}\right\} .
$$

If $g_{0} \in \Delta$ and if $Y_{g_{0}}$ has but one singular point and if it is non-degenerate quadratic, then there is associated to it special monodromies (Picard-Lefschetz transformations)

$$
T v=v-(v, a) a
$$

where $a \in H_{n-s}\left(Y_{0}, \mathbf{C}\right)$ is called a vanishing cycle, and $($,$) is the middle$ homology pairing.

We define

van $=$ span of the vanishing cycles determined by the family $\eta$.

The next theorem is a main ingredient of the Second Lefschetz Theorem.

(5.2) Theorem. Let $Z^{r}$ be a rigged homogeneous space with group $G^{N}$ (cf. §3). Let $Y^{r-s}$ be a compact smooth connected subvariety of $Z$ with an ample normal bundle, and let $X$ be a smooth connected closed subvariety of $Z$ such that $\mathbf{P}\left(N^{*} X\right)$ meets the open orbit $O_{m}$. cf (4.5.2).

Then there is a smooth connected open subvariety, $D$, of the discriminant $\Delta^{N-1}$ such that

(i) $\operatorname{dim}(\Delta \backslash D) \leq N-2$, and

(ii) if $g \in D$ then $Y_{g}$ has only one singular point and it is nondegenerate quadratic. 
REMARK 1. If $\operatorname{dim} \Delta \leq N-2$ the conclusion is trivially satisfied by taking $D$ to be the empty set.

Remark 2. The hypothesis that $\mathbf{P}\left(N^{*} X\right)$ should meet the open orbit $O_{m}$ is stronger than necessary. For example, if $Z=\operatorname{Gr}\left(2, \mathbf{C}^{2 k}\right)$ and $Y$ is a smooth hyperplane section of $Z$, then $\mathbf{P}\left(N^{*} Y\right) \subset O_{m}$. Thus, if $\mathbf{P}\left(N^{*} X\right) \cap$ $O_{m}=\varnothing$ then the discriminant $\Delta$ is empty.

Proof of theorem. Construct the linear fibre space $\mathcal{E}$, as in (4.1).

There is a diagram

$$
\varepsilon \rightarrow \quad \eta \stackrel{p}{\rightarrow} \quad G
$$

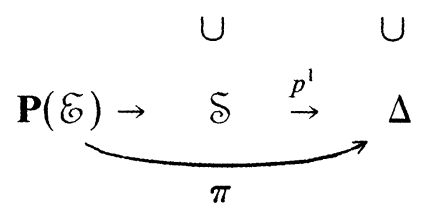

(The notation is described in (5.1).)

Recall (4.4) that $\mathbf{P}(\mathscr{E})=\cup_{\rho} \mathbf{P}(\mathcal{E})_{\rho}$. Let

$$
\begin{aligned}
& E_{1}=\pi\left(\bigcup_{\rho \neq m} \mathbf{P}(\mathscr{E})_{\rho}\right), \\
& E_{2}=p^{1}\left\{(x, g): \operatorname{rk}_{(x, g)} p^{1} \leq N-2\right\}, \\
& E_{3}=\left\{g \in \Delta: \operatorname{dim}\left(\bigcap_{x \in Y_{g}} \operatorname{im}\left(p_{*}\right)\right) \leq N-2\right\} .
\end{aligned}
$$

Put $D=\Delta \backslash\left(E_{1} \cup \bar{E}_{2} \cup \bar{E}_{3} \cup \operatorname{sing}(\Delta)\right)$.

Certainly, $D$ is a smooth open subset of $\Delta$, and is also connected since $D$ is an open subset of the irreducible variety $\pi\left(\overline{\mathbf{P}(\mathcal{E})_{m}}\right) \mathrm{cf}$. (4.7).

By Corollary (4.5.3), $\operatorname{dim}\left(E_{1}\right) \leq N-2$, and by Corollary (1.7.1) we also have

$$
\operatorname{dim}\left(E_{2}\right) \leq N-2 \text { and } \operatorname{dim}\left(E_{3}\right) \leq N-2 .
$$

Thus, $\operatorname{dim}(\Delta \backslash D) \leq N-2$.

The restricted mapping

$$
\left.\mathbf{P}(\mathcal{E})\right|_{\delta_{1}} \rightarrow \mathcal{S}_{1}
$$


is a bijection of sets, as is easily seen from (4.1.3). In fact, it is an isomorphism of varieties. This is a local calculation using the special coordinates of [6] Lemma (3.3). From Proposition (4.7) it, now, follows that $\mathfrak{S}_{1}$ is smooth $(N-1$ dimensional $)$ at each point over $D$.

Fix, now, a point $g \in D$.

As in [6] Proposition (3.3.3), each singular point of $Y_{g}$ is nondegenerate quadratic since $g \notin E_{2}$.

Moreover, since $g \notin E_{3}$, we have

$$
\operatorname{dim}\left(\sum_{x \in Y_{g}} \operatorname{ker} p_{(x, g)}^{*}\right)=1 .
$$

We remark that $\operatorname{ker} p^{*}=\left(\operatorname{im} p_{*}\right)^{\perp}$.

Now, if $x_{1}$ and $x_{2}$ are two singular points of $Y_{g}$ then

$$
\operatorname{ker} p_{\left(x_{1}, g\right)}^{*}=\operatorname{ker} p_{\left(x_{2}, g\right)}^{*}
$$

as elements of $\mathbf{P}\left(T_{g}^{*}(G)\right)$. Referring to diagram (4.3.1), we see that $x_{1}=x_{2}$ since $\pi^{-1}(D) \subset \mathbf{P}(\mathcal{E})_{m}$ and $\phi_{\mathscr{E}}$ is $1-1$ on $\mathbf{P}(\mathcal{E})_{m}$ (cf. (3.1)iv).

(5.3) THEOREM (Second Lefschetz Theorem). Let $Z$ be a Kaehler complex algebraic rigged homogeneous space (e.g. Grassmanian, quadric, $\mathbf{P}^{r} \backslash \mathbf{P}^{k}$ cf. §3). Let $Y$ be a connected smooth compact subvariety of $Z$ with an ample normal bundle $N Y$. Let $X$ be a connected smooth closed subvariety of $Z$ such that $\mathbf{P}\left(N^{*} X\right)$ meets the open orbit of $\mathbf{P}\left(T^{*} Z\right)$ cf. (2.4) and Corollary (4.5.2).

Let $\operatorname{dim} Z=r, \operatorname{dim} X=n$ and $\operatorname{dim} Y=r-s$. Assume that $n+s \leq$ $r+1,2 s \leq r$ and $\pi_{i}(Z, Y)=0$ for $i \leq n-s+1$. (This last assumption is satisfied, for example, when $n+s \leq r$ (5.0.1).)

Let $Y_{0}=X \cap Y$ and assume that $X$ meets $Y$ transversely in $Z$. In particular, $Y_{0}$ is a smooth $n-s$ dimensional subvariety of $X$. Let

ker $=$ kernel of $H_{n-s}\left(Y_{0}, \mathbf{C}\right) \rightarrow H_{n-s}(X, \mathbf{C})$,

van $=$ span of vanishing cycles in $H_{n-s}\left(Y_{0}, \mathrm{C}\right)$, and

inv $=$ cycles in $H_{n-s}\left(Y_{0}, \mathbf{C}\right)$ invariant under the monodromy cf. (5.1.1). Then

$H_{n-s}\left(Y_{0}, \mathbf{C}\right)=\operatorname{ker} \oplus \operatorname{inv}$,

ker $=$ van,

the monodromy acts transitively, up to sign, on the set of vanishing cycles, and

van is irreducible under the monodromy action. 
There is a corresponding conclusion in cohomology.

Proof. The proof is essentially the same as that of [6] (6.1). In the latter, replace " $\mathbf{P}^{r}$ " by " $Z$ " and [6] Theorem (3.5) by Theorem (5.2) of this paper.

We need, now, only two lemmas to modify the proof of [6] (6.1)ii.

Let $\nu: G^{1} \rightarrow G$ be the universal cover of $G$, which is an algebraic map (3.1i). Put $\eta^{1}=\nu^{-1}(\eta)$, cf. (1.10).

Lemma. $H^{n-s}\left(X \times G^{1}, \mathbf{C}\right) \rightarrow H^{n-s}\left(\eta^{1}, \mathbf{C}\right)$ is onto.

Proof. The map

$$
\begin{aligned}
& X \times G^{1} \rightarrow Z, \\
& \quad(x, g) \rightarrow \nu(g) x
\end{aligned}
$$

is a fibration, so the proof of [6] (6.1.3) applies.

LEMMA. $\operatorname{van}^{\perp} \subset$ inv.

Proof. We show, in fact, that $\operatorname{van}^{\perp} \subset$ im. Since $\pi_{1}\left(G^{1}\right)=0$, the monodromy subgroup, $\Re^{1}$, of $\operatorname{Aut}\left(H_{n-s}\left(Y_{0}, \mathbf{C}\right)\right)$ determined by the family $\eta^{1}$ is generated by the Picard-Lefschetz transformations cf. (5.1.2). This implies that $\operatorname{van}^{\perp}=$ inv $^{1}$, where

$$
\operatorname{inv}^{1}\left\{\alpha \in H_{n-s}\left(Y_{0}, \mathbf{C}\right): T \alpha=\alpha \forall T \in \Re^{1}\right\} .
$$

But inv $^{1}=\mathrm{im}$, as in [6], (6.1)iii.

6. Concluding remarks. The question in [6] $\S 7$ asks whether there is a smooth hypersurface $Y$ in $\operatorname{Gr}(2,4)$ such that $\mathbf{P}\left(N^{*} Y\right)$ is contained in the lower dimensional orbit of rank 1 vectors. According to Proposition (6.5) this is impossible, since $N Y$ is necessarily ample. Actually, Andrew Sommese had answered the above question using a line bundle argument, and this was encouragement enough to work out a general solution.

Note added in proof. In Definition 3.1, condition (ii) may be replaced by the weaker condition "The action of $G$ on $\mathbf{P}\left(T^{*} Z\right)$ has an open orbit, $O_{m}$ " i.e. one need not require that there be finitely many orbits. In "Finding the nondegenerate quadratic singularities", Proc. Symp. Pure Math., 40 (1982), the author indicates why this is so. 


\section{REFERENCES}

[1] A. Andreotti and T. Frankel, The Lefschetz theorem on hyperplane sections, Ann. of Math., 69 (1959), 713-717.

[2] W. Barth, Larsen's theorem on the homotopy groups of projective manifolds of small embedding codimension, Proc. Symp. Pure Math., 29 (1975), 307-313.

[2A] _ Transplanting cohomology classes, Amer. J. Math., 92 (1970), 951-967.

[3] C. Chevally, Classification de groupes de Lie algébriques, Sem. C. Chevalley. Ecole Norm. Sup. 1956-58. Vol. 1, Ch. 8.

[4] Fondemonts de la géométrie algébrique, Paris: Secrétariat mathématique 1958.

[5] G. Fischer, Complex analytic geometry, Lecture Notes in Mathematics, Vol. 538, Berlin, Heidelberg, New York, Springer 1976.

[6] N. Goldstein, A Second Lefschetz Theorem for general manifold sections in complex projective space, Math. Ann., 246 (1979), 41-68.

[7] _ Families of varieties and a Second Lefschetz Theorem for manifold sections in complex projective space, Thesis. Cornell University, 1979.

[8] R. Gunning and H. Rossi, Analytic Functions of Several Complex Variables, Englewood Cliffs, NH: Prentice-Hall, 1965.

[9] R. Hartshorne, Ample subvarieties of algebraic varieties, Lecture Notes in Mathematics, 156. Springer-Verlag, Berlin, Heidleberg, New York, 1970.

[10] __ Ample vector bundles, Publ. Math. I.H.E.S., 29 (1966), 63-94.

[11] W. V. D. Hodge and D. Pedoe, Methods of algebraic geometry, vols. I, II, III. Cambridge University Press, 1952.

[12] M. E. Larsen, On the topology of complex projective manifolds, Invent. Math., 19 (1973), 251-260.

[13] S. Lefschetz, L'analysis situs et la géometrie algébrique, Paris: Gauthier-Villars, 1924.

[14] A. Papantonopoulou, Curves in Grassman varieties, Nagoya Math. J., 66 (1977), 121-137.

[15] E. Robinson, A characterization of certain branched coverings as group actions, Fund. Math. CIII.1 (1979), 43-45.

[16] I. R. Shafarevich, Basic Algebraic Geometry, Berlin, Heidelberg, New York: Springer-Verlag, 1974.

[17] A. J. Sommese, Submanifolds of Abelian varieties, Math. Ann., 233 (1978), 229-256.

[18] __, Theorems of Barth-Lefschetz type for complex subspaces of homogeneous complex manifolds, Proc. Natl. Acad. Sci., 74 (1977), 1332-1333.

[19] Complex subspaces of homogeneous complex manifolds I, Duke J., 46 (1979), $527-548$.

[20] Complex subspaces of homogeneous complex manifolds II, Nagoya Mathematical J. To appear.

Received July 2, 1981.

UNIVERSITY OF BRITISH COLUMBIA

VANCUUVER, BC

CANADA V6T 1Y4

Current address: Purdue University

West Lafayette, IN 47907 



\title{
PACIFIC JOURNAL OF MATHEMATICS \\ EDITORS
}

\author{
Donald BABBITT (Managing Editor) \\ University of California \\ Los Angeles, CA 90024 \\ Hugo Rossi \\ University of Utah \\ Salt Lake City, UT 84112 \\ C. C. MOore and ARTHur OGus \\ University of California \\ Berkeley, CA 94720
}

\author{
J. Dugundi \\ Department of Mathematics \\ University of Southern California \\ Los Angeles, CA 90089-1113
}

R. FINN and H. SAMELSON

Stanford University

Stanford, CA 94305

ASSOCIATE EDITORS
R. ARENS
E. F. BECKENBACH
B. H. NeUmanN
F. WOLF
K. YosHidA
(1906-1982)

\section{SUPPORTING INSTITUTIONS}

\author{
UNIVERSITY OF ARIZONA \\ UNIVERSITY OF BRITISH COLUMBIA \\ CALIFORNIA INSTITUTE OF TECHNOLOGY \\ UNIVERSITY OF CALIFORNIA \\ MONTANA STATE UNIVERSITY \\ UNIVERSITY OF NEVADA, RENO \\ NEW MEXICO STATE UNIVERSITY \\ OREGON STATE UNIVERSITY
}

\author{
UNIVERSITY OF OREGON \\ UNIVERSITY OF SOUTHERN CALIFORNIA \\ STANFORD UNIVERSITY \\ UNIVERSITY OF HAWAII \\ UNIVERSITY OF TOKYO \\ UNIVERSITY OF UTAH \\ WASHINGTON STATE UNIVERSITY \\ UNIVERSITY OF WASHINGTON
}

The Supporting Institutions listed above contribute to the cost of publication of this Journal, but they are not owners or publishers and have no responsibility for its content or policies.

Mathematical papers intended for publication in the Pacific Journal of Mathematics should be in typed form or offset-reproduced (not dittoed), double spaced with large margins. Please do not use built up fractions in the text of the manuscript. However, you may use them in the displayed equations. Underline Greek letters in red, German in green, and script in blue. The first paragraph must be capable of being used separately as a synopsis of the entire paper. In particular it should contain no bibliographic references. Please propose a heading for the odd numbered pages of less than 35 characters. Manuscripts, in triplicate, may be sent to any one of the editors. Please classify according to the scheme of Math. Reviews, Index to Vol. 39. Supply name and address of author to whom proofs should be sent. All other communications should be addressed to the managing editor, or Elaine Barth, University of California, Los Angeles, California 90024.

There are page-charges associated with articles appearing in the Pacific Journal of Mathematics. These charges are expected to be paid by the author's University, Government Agency or Company. If the author or authors do not have access to such Institutional support these charges are waived. Single authors will receive 50 free reprints; joint authors will receive a total of 100 free reprints. Additional copies may be obtained at cost in multiples of 50 .

The Pacific Journal of Mathematics is issued monthly as of January 1966. Regular subscription rate: $\$ 132.00$ a year (6 Vol., 12 issues). Special rate: $\$ 66.00$ a year to individual members of supporting institutions.

Subscriptions, orders for numbers issued in the last three calendar years, and changes of address should be sent to Pacific Journal of Mathematics, P.O. Box 969, Carmel Valley, CA 93924, U.S.A. Old back numbers obtainable from Kraus Periodicals Co., Route 100, Millwood, NY 10546.

The Pacific Journal of Mathematics ISSN 0030-8730 is published monthly by the Pacific Journal of Mathematics at P.O. Box 969, Carmel Valley, CA 93924. Application to mail at Second-class postage rates is pending at Carmel Valley, California, and additional mailing offices. Postmaster: Send address changes to Pacific Journal of Mathematics, P. O. Box 969, Carmel Valley, CA 93924.

PUBLISHED BY PACIFIC JOURNAL OF MATHEMATICS, A NON-PROFIT CORPORATION

Copyright $(1983$ by Pacific Journal of Mathematics 


\section{Pacific Journal of Mathematics}

Vol. 106, No. 2 December, 1983

R. J. Gazik, Non-Hausdorff convergence spaces $\ldots \ldots \ldots \ldots \ldots \ldots \ldots 257$

John Norman Ginsburg, A note on the cardinality of infinite partially

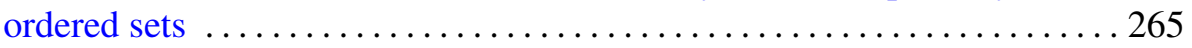

Norman Joseph Goldstein, Ampleness in complex homogeneous spaces

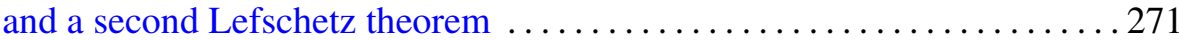

Clyde D. Hardin and Loren Dallas Pitt, Integral invariants of functions and

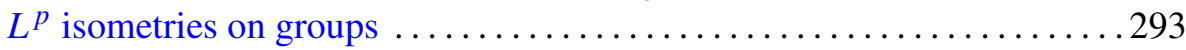

M. Scott Osborne and Garth William Warner, Jr., The Selberg trace formula. II. Partition, reduction, truncation 307 